\title{
On the Stability Analysis of Rational Integrator Method for the Solution of Initial Value Problems in Ordinary Differential Equation
}

\author{
Agbeboh Goddy Ujagbe ${ }^{1}$, Ehiemua Michael Ebhodaghe ${ }^{1, ~ *}$, Loko Perelah ${ }^{2}$ \\ ${ }^{1}$ Department of Mathematics, Ambrose Alli Unuversity, Ekpoma, Nigeria \\ ${ }^{3}$ Department of Mathematics, Bayelsa State College of Education, Sagbama, Nigeria
}

Email address:

Agbebohgoddy@gmail.com (A. G. Ujagbe),mikkyoneehi@gmail.com (E. M. Ebhodaghe), perelahloko@gmail.com (L. Perelah)

${ }^{*}$ Corresponding author

\section{To cite this article:}

Agbeboh Goddy Ujagbe, Ehiemua Michael Ebhodaghe, Loko Perelah. On the Stability Analysis of Rational Integrator Method for the Solution of Initial Value Problems in Ordinary Differential Equation. American Journal of Mathematical and Computer Modelling.

Vol. 5, No. 4, 2020, pp. 102-108. doi: 10.11648/j.ajmcm.20200504.12

Received: September 27, 2020; Accepted: October 15, 2020; Published: October 23, 2020

\begin{abstract}
In all numerical methods, it is necessary to ascertain the validity of any particular scheme. And this is possible to determine, by verifying the nature of the stability of that scheme. So the general stability function definition is given, from where an investigation is carried out on a class of rational integrator of order 15 , to establish the region of absolute stability of the scheme, by constructing the Jordan curve. In the process of expanding the rational function, binomial theorem as well as the idea of combination process were introduced to ease the computation by using Maple-18 package. The simplification of the general rational integrator formula, is constructed from two processes namely through complex function, and then through polar analysis, The Jordan curve is constructed with the help of MATLAB package. Furthermore, it was discovered that the region of instability is on the positive side of the complex plane, while the region of absolute stability is outside the Jordan curve. Finally, it is further established that the encroachment point, $\tau$, lie within the interval \pm 140.6 . And the encroachment point is visible from the corresponding values of $\varnothing$ and $\mathrm{R}$ at the extremes. The stability curve revealed that the integrator is not only A-stable, but also Lstable.
\end{abstract}

Keywords: Rational Integrator, Encroachment Point, Binomial Theorem, Absolute Stability, Jordan Curve, A-stable, L-Stability

\section{Introduction}

Numerical methods for evaluating systems of Ordinary Differential Equations (ODEs) have been attracting much attention because they proffer the solutions of problems arising from the mathematical formulation of physical situations such as those in chemical kinetics, population, economic, political and social models. Numerical solution of ordinary differential equations can be obtained using rational integrators, such as linear multistep, Runge-Kutta and exponential methods and many others. There are quite a number of stiff problems that can be solved by rational integrators, which are from physical reactions, chemical kinetic and life sciences. The object of our study is the stability function of a general rational integrator reported in
Aashikpelokhai [1] and whose underlying interpolant is a rational function, $P_{L}(x) \cdot\left(Q_{M}(x)\right)^{-1}$ where $P_{L}(x)$ and $Q_{M}(x)$ are polynomial functions of degrees $\mathrm{L}$ and $\mathrm{M}$ respectively. The stability function of any integrator is what is normally used in determining the Region of Absolute Stability of such an integrator. A common yardstick which is used in the determination of the Region of Absolute Stability (RAS) is the unit ball in $R^{n}$. The RAS is the same as the region in which the absolute value of the stability function lies in the unit ball in $R^{n}$. The works of Aashikpelokhai [1], Aashikpelokhai and Momodu [2], Elakhe, Onianwa and Elakhe [3], Agbeboh [4] and Abhulimen and Otunta [5], established that rational integrator methods, can solve these problems. Agbeboh and Aashikpelokhai [6] worked on implementation of rational integrator, of order 26 , which produced result that compared 
favorably well with other existing methods without really establishing the stability region of the method. All of these works were basically for the improvement of numerical solutions to initial value problems, which sets the foundation for the study. The rational interpolating method is used to find the numerical establishment of the stability function of a general rational integrator, reported in Aashikpelokhai [1], with particular attention to the case $\mathrm{k}=8$ and show the region of Absolute stability of the integrator. The region of absolute stability (RAS), is the same as the region in which the absolute value of the stability function lies in the unit circle. As Aashikpelokhai [1] puts it, the smallness of the individual error is called accuracy but the ability to keep the effect of this error under control is called stability. He further opined that the region of absolute stability (RAS) of rational interpolation methods always lie entirely on the left-half of the complex plane. Therefore, for a rational interpolation method to be useful, it should possess an appropriate stability property. Hence the overall aim of this study is to determine the stability polynomial, and establish the region of absolute stability of a rational integrator of order 15 , that is when $\mathrm{k}=8$. Our computational experience as exemplified by the works of Aashikpelokhai and Elakhe [7] along with the research work given by Islam [8], Agbeboh and Esekhaigbe [9], Elakhe and Aashikpelokhai [10], Fatunla and Aashikpelokhai [11], Elakhe, Aashikpelokhai and Ebhomien [12], Awari [13], Momodu [14] and Fatunla [15], all give credence to the need for rational integrators. Basically, there are two ways of approaching the expansion of the stability function; these may be through direct algebraic method using $\mathrm{F}(\mathrm{u}, \mathrm{v})$ or using polar method of $\mathrm{F}(\mathrm{R}, \phi)$. By employing the binomial expansion on $\mathrm{F}(\mathrm{u}, \mathrm{v})$, we established the complex integrating function and reduce the expansion to polar form at which point we introduce $\mathrm{F}(\mathrm{R}, \phi)$, where $\mathrm{R}$ represents the polynomial of the complex integrator function, while argument $(\phi)$ is the angle of rotation, about which the roots of $\mathrm{R}$, are determined. After the expansion, the integrator is subjected to a sequence of solvability analysis to establish the stability function for the rational integrator for the case $\mathrm{k}=8$. The roots of the complex polynomial are determined to enable the plotting of the Jordan curve, which will show both the Region of Absolute stability (RAS) and the Region of instability (RIS)

\section{Analysis of the Stability Function}

The general rational integration method, according to Aashikpelokhai [1], is given as:

$$
y_{n+1}=\frac{\sum_{r=0}^{k-1} p_{r} x_{n+1}^{r}}{1+\sum_{r=0}^{k} q_{r} x_{n+1}^{r}}
$$

Where $M=k$ and $L=k-1$

But the general stability function of the rational integrator scheme is defined as:

$$
S(\bar{h})=\frac{\sum_{r=0}^{k-1}(2 k-1-i) !\left(\begin{array}{c}
k-1 \\
i
\end{array}\right) \bar{h}^{r}}{\sum_{r=0}^{k}(-1)^{r}(2 k-1-i) !\left(\begin{array}{c}
k \\
i
\end{array}\right) \bar{h}^{r}}
$$

Where

$$
\begin{gathered}
\theta(\bar{h})=\sum_{r=0}^{k-1}(2 k-1-i) !\left(\begin{array}{c}
k-1 \\
i
\end{array}\right) \bar{h}^{r} \\
\psi(\bar{h})=\sum_{r=0}^{k}(-1)^{r}(2 k-1-i) !\left(\begin{array}{l}
k \\
i
\end{array}\right) \bar{h}^{r} \\
S(\bar{h})=\frac{\theta(\bar{h})}{\psi(\bar{h})}<1
\end{gathered}
$$

By a process of binomial expansion, we expand the numerator and the denominator of (2) above.

Here (3) is expanded with $\mathrm{k}=8$, to get

$$
\begin{aligned}
\theta(h)= & 40320 \bar{h}^{7}+2540160 \bar{h}^{6}+76204800 \bar{h}^{5} \\
& +1397088000 \bar{h}^{4}+16765056000 \bar{h}^{3} \\
& +130767436800 \bar{h}^{2}+610248038400 \bar{h} \\
& +13077674368000
\end{aligned}
$$

And (4) gives

$$
\begin{aligned}
\psi(\bar{h})= & 5040 \bar{h}^{8}-322560 \bar{h}^{7}+10160640 \bar{h}^{6}-203212800 \bar{h}^{5} \\
& +2794176000 \bar{h}^{4}-26824089600 \bar{h}^{3}+174356582400 \bar{h}^{2} \\
& -697426329600 h+1307674368000
\end{aligned}
$$

Now letting $\bar{h}=u+i v$, where $i^{2}=-1$, then (6) becomes:

$$
\begin{aligned}
\theta(\bar{h})= & 40320(i v+u)^{7}+2540160(i v+u)^{6} \\
& +76204800(i v+u)^{5}+1397088000(i v+u)^{4} \\
& +16765056000(i v+u)^{3}+130767436800(i v+u)^{2} \\
& +610248038400(i v+u)+1307674368000
\end{aligned}
$$

Separating (8) in the form $(A+i B)$, we have;

$$
\begin{aligned}
A= & v^{6}+2540160 u^{6}-38102400 u^{4} v^{2}+38102400 u^{2} v^{4} \\
& -2540160 v^{6}+76204800 u^{5}-762048000 u^{3} v^{2} \\
& +381024000 u v^{4}+1397088000 u^{4}-8382528000 u^{2} v^{2} \\
& +1397088000 v^{4}+16765056000 u^{3}-50295168000 u v^{2} \\
& +130767436800 u^{2}-130767436800 v^{2}+610248038400 u \\
& +1307674368000
\end{aligned}
$$




$$
\begin{gathered}
B=282240 u^{6} v-1411200 u^{4} v^{3}+846720 u^{2} v^{5} \\
-40320 v^{7}+15240960 u^{5} v-50803200 u^{3} v^{3} \\
+15240960 u v^{5}+381024000 u^{4} v-762048000 u^{2} v^{3} \\
+76204800 v^{5}+5588352000 u^{3} v-5588352000 u v^{3} \\
+50295168000 u^{2} v-16765056000 v^{3} \\
+261534873600 u v+610248038400 v \\
\psi(\bar{h})= \\
-2040(u+i v)^{8}-322560(u+i v)^{7}+10160640(u+i v)^{6} \\
-203212800(u+i v)^{5}+2794176000(u+i v)^{4} \\
-26824089600(u+i v)^{3}+174356582400(u+i v)^{2} \\
-
\end{gathered}
$$

Separating (11) in the form $(C+i D)$, we have;

$$
\begin{aligned}
C= & 5040 u^{8}-141120 u^{6} \mathrm{v}^{2}+352800 u^{4} \mathrm{v}^{4}-141120 u^{2} \mathrm{v}^{6} \\
& +5040 v^{8}-322560 u^{7}+6773760 u^{5} \mathrm{v}^{2}-11289600 u^{3} \mathrm{v}^{4} \\
& +2257920 u v^{6}+10160640 u^{6}-152409600 u^{4} \mathrm{v}^{2} \\
& +152409600 u^{2} \mathrm{v}^{4}-10160640 v^{6}-203212800 u^{5} \\
& +2032128000 u^{3} \mathrm{v}^{2}-1016064000 u v^{4}+2794176000 u^{4} \\
& -16765056000 u^{2} \mathrm{v}^{2}+2794176000 v^{4}-26824089600 u^{3} \\
& +80472268800 u v^{2}+174356582400 u^{2}-174356582400 v^{2} \\
& -697426329600 u+1307674368000
\end{aligned}
$$

and

$$
\begin{aligned}
D= & 40320 u^{7} \mathrm{v}-282240 u^{5} \mathrm{v}^{3}+282240 u^{3} \mathrm{v}^{5} \\
& -40320 u v^{7}-2257920 u^{6} \mathrm{v}+11289600 u^{4} \mathrm{v}^{3} \\
& -6773760 u^{2} \mathrm{v}^{5}+322560 v^{7}+60963840 u^{5} \mathrm{v} \\
& -203212800 u^{3} \mathrm{v}^{3}+60963840 u v^{5}-1016064000 u^{4} \mathrm{v} \\
& +2032128000 u^{2} \mathrm{v}^{3}-203212800 v^{5}+11176704000 u^{3} \mathrm{v} \\
& -11176704000 u v^{3}-80472268800 u^{2} \mathrm{v}+26824089600 v^{3} \\
& +348713164800 u v-697426329600 v
\end{aligned}
$$

From (5), we have that:

$S(\bar{h})=\frac{\theta(\bar{h})}{\psi(\bar{h})}<1$ if and only if:

$$
\left.\begin{array}{l}
|\theta(\bar{h})|^{2}<|\psi(\bar{h})|^{2} \\
|\theta(\bar{h})|^{2}-|\psi(\bar{h})|^{2}<0
\end{array}\right\}
$$

Therefore, computing $\mathrm{A}^{2}$ we have: 


$$
\left(\begin{array}{c}
v^{6}+2540160 u^{6}-38102400 u^{4} v^{2}+38102400 u^{2} v^{4}-2540160 v^{6}+76204800 u^{5}-762048000 u^{3} v^{2} \\
+381024000 u v^{4}+1397088000 u^{4}-8382528000 u^{2} v^{2}+1397088000 v^{4}+16765056000 u^{3} \\
-50295168000 u v^{2}+130767436800 u^{2}-130767436800 v^{2}+610248038400 u+1307674368000
\end{array}\right)^{2}
$$

Then $\mathrm{B}^{2}$ becomes

$$
\left(\begin{array}{c}
282240 u^{6} \mathrm{v}-1411200 u^{4} \mathrm{v}^{3}+846720 u^{2} \mathrm{v}^{5}-40320 v^{7}+15240960 u^{5} \mathrm{v}-50803200 u^{3} \mathrm{v}^{3} \\
\quad+15240960 u v^{5}+381024000 u^{4} \mathrm{v}-762048000 u^{2} \mathrm{v}^{3}+76204800 v^{5}+5588352000 u^{3} \mathrm{v} \\
-5588352000 u v^{3}+50295168000 u^{2} \mathrm{v}-16765056000 v^{3}+261534873600 u v \\
+610248038400 v
\end{array}\right)^{2}
$$

Therefore

$$
\left.\begin{array}{rl}
\left(\mathrm{A}^{2}+\mathrm{B}^{2}\right)=\left(\begin{array}{c}
v^{6}+2540160 u^{6}-38102400 u^{4} \mathrm{v}^{2}+38102400 u^{2} \mathrm{v}^{4}-2540160 v^{6}+76204800 u^{5}-762048000 u^{3} \mathrm{v}^{2} \\
+381024000 u v^{4}+1397088000 u^{4}-8382528000 u^{2} \mathrm{v}^{2}+1397088000 v^{4}+16765056000 u^{3} \\
-50295168000 u v^{2}+130767436800 u^{2}-130767436800 v^{2}+610248038400 u+1307674368000
\end{array}\right.
\end{array}\right)^{2}
$$

Similarly

$$
C^{2}=\left(\begin{array}{c}
5040 u^{8}-141120 u^{6} \mathrm{v}^{2}+352800 u^{4} \mathrm{v}^{4}-141120 u^{2} \mathrm{v}^{6} \\
+5040 v^{8}-322560 u^{7}+6773760 u^{5} \mathrm{v}^{2}-11289600 u^{3} \mathrm{v}^{4} \\
+2257920 u v^{6}+10160640 u^{6}-152409600 u^{4} \mathrm{v}^{2} \\
+152409600 u^{2} \mathrm{v}^{4}-10160640 v^{6}-203212800 u^{5} \\
+2032128000 u^{3} v^{2}-1016064000 u v^{4}+2794176000 u^{4} \\
-16765056000 u^{2} v^{2}+2794176000 v^{4}-26824089600 u^{3} \\
+80472268800 u v^{2}+174356582400 u^{2}-174356582400 v^{2} \\
-697426329600 u+1307674368000
\end{array}\right)^{2}
$$

and

$$
\mathrm{D}^{2}=\left(\begin{array}{c}
40320 u^{7} \mathrm{v}-282240 u^{5} \mathrm{v}^{3}+282240 u^{3} \mathrm{v}^{5}-40320 u v^{7}-2257920 u^{6} \mathrm{v} \\
+11289600 u^{4} \mathrm{v}^{3}-6773760 u^{2} \mathrm{v}^{5}+322560 v^{7}+60963840 u^{5} \mathrm{v} \\
-203212800 u^{3} \mathrm{v}^{3}+60963840 u v^{5}-1016064000 u^{4} \mathrm{v}+2032128000 u^{2} \mathrm{v}^{3} \\
-203212800 v^{5}+11176704000 u^{3} \mathrm{v}-11176704000 u v^{3}-80472268800 u^{2} \mathrm{v} \\
+26824089600 v^{3}+348713164800 u v-697426329600 v
\end{array}\right)^{2}=
$$




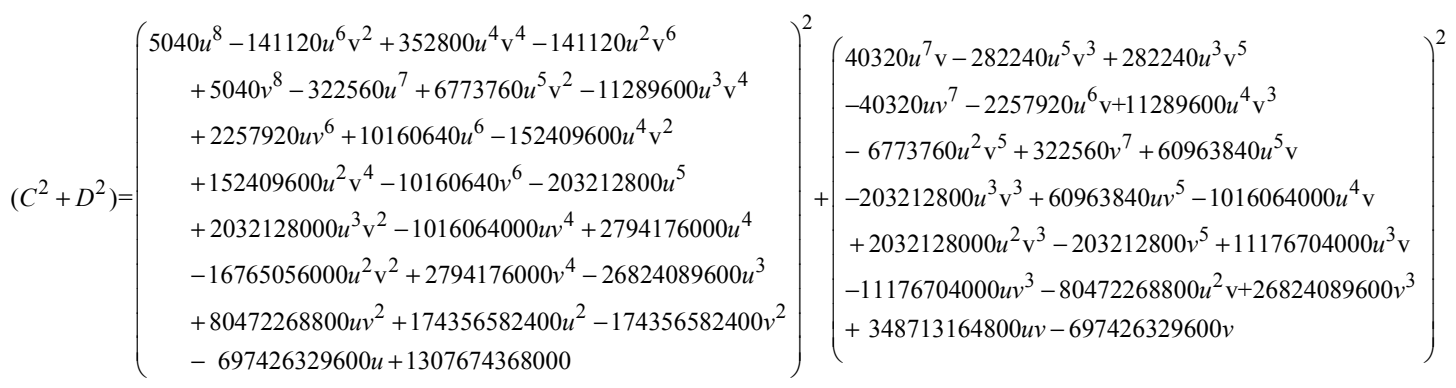

Then to compute $\left(\mathrm{A}^{2}+\mathrm{B}^{2}\right)-\left(\mathrm{C}^{2}+\mathrm{D}^{2}\right)$, we get:

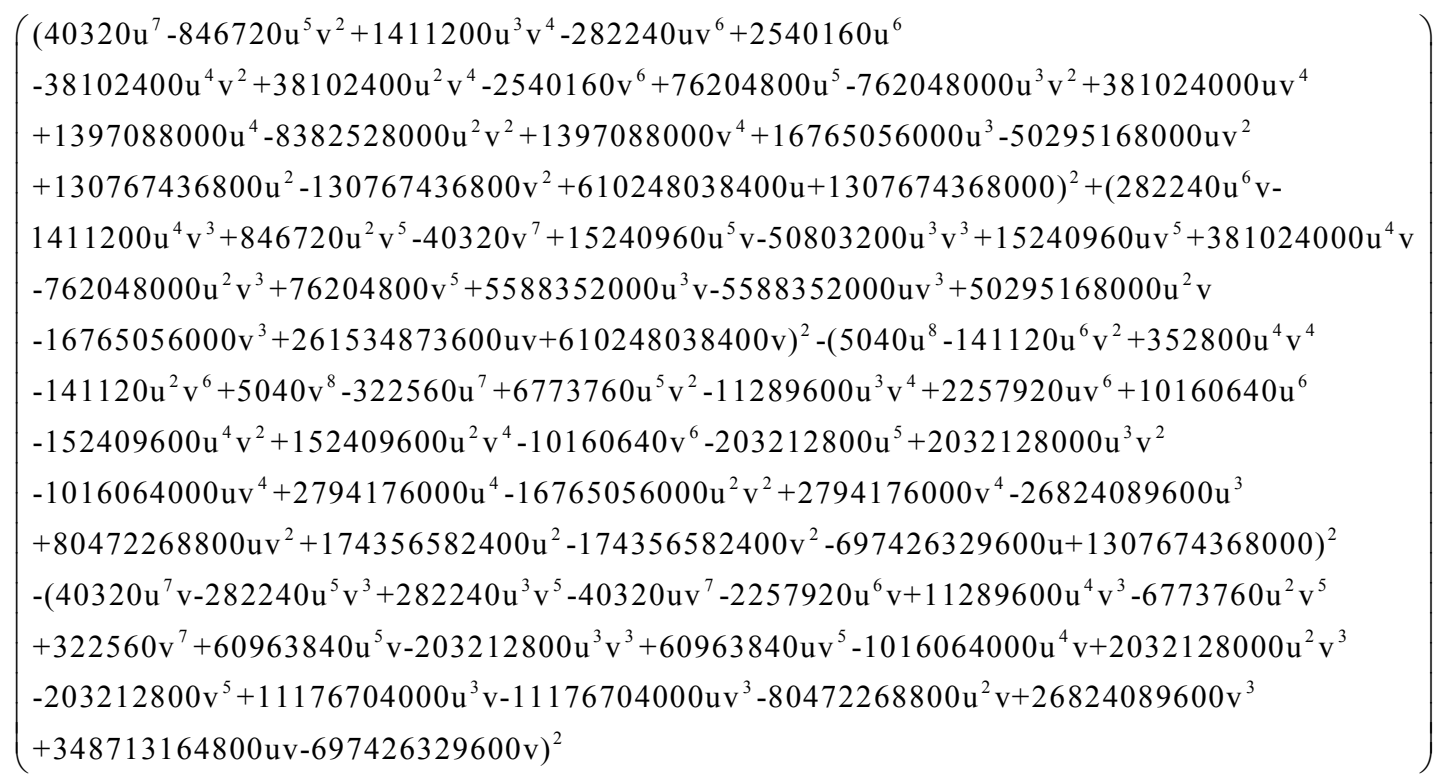

At this point we need to convert (22) into the polar form $F(R, \phi)$. The only way to do this is to take $u=R \cos (\varnothing)$ and $v=R$ $\sin (\varnothing)$, and then $(22)$ becomes:

$\left(\left(40320 \mathrm{R}^{7} \cos (\phi)^{7}-846720 \mathrm{R}^{7} \cos (\phi)^{5} \sin (\phi)^{2}+1411200 \mathrm{R}^{7} \cos (\phi)^{3} \sin (\phi)^{4}-282240 \mathrm{R}^{7} \cos (\phi) \sin (\phi)^{6}\right.\right.$

$+2540160 \mathrm{R}^{6} \cos (\phi)^{6}-38102400 \mathrm{R}^{6} \cos (\phi)^{4} \sin (\phi)^{2}+38102400 \mathrm{R}^{6} \cos (\phi)^{2} \sin (\phi)^{4}-2540160 \mathrm{R}^{6} \sin (\phi)^{6}$

$+76204800 \mathrm{R}^{5} \cos (\phi)^{5}-762048000 \mathrm{R}^{5} \cos (\phi)^{3} \sin (\phi)^{2}+381024000 \mathrm{R}^{5} \cos (\phi) \sin (\phi)^{4}+1397088000 \mathrm{R}^{4} \cos (\phi)^{4}$

$-8382528000 \mathrm{R}^{4} \cos (\phi)^{2} \sin (\phi)^{2}+1397088000 \mathrm{R}^{4} \sin (\phi)^{4}+16765056000 \mathrm{R}^{3} \cos (\phi)^{3}-50295168000 \mathrm{R}^{3} \cos (\phi) \sin (\phi)^{2}$

$\left.+130767436800 \mathrm{R}^{2} \cos (\phi)^{2}-130767436800 \mathrm{R}^{2} \sin (\phi)^{2}+610248038400 \mathrm{R} \cos (\phi)+1307674368000\right)^{2}$

$+\left(282240 \mathrm{R}^{7} \cos (\phi)^{6} \sin (\phi)-1411200 \mathrm{R}^{7} \cos (\phi)^{4} \sin (\phi)^{3}+846720 \mathrm{R}^{7} \cos (\phi)^{2} \sin (\phi)^{5}-40320 \mathrm{R}^{7} \sin (\phi)^{7}\right.$

$+15240960 \mathrm{R}^{6} \cos (\phi)^{5} \sin (\phi)-50803200 \mathrm{R}^{6} \cos (\phi)^{3} \sin (\phi)^{3}+15240960 \mathrm{R}^{6} \cos (\phi) \sin (\phi)^{5}+381024000 \mathrm{R}^{5} \cos (\phi)^{4} \sin (\phi)$

$-762048000 \mathrm{R}^{5} \cos (\phi)^{2} \sin (\phi)^{3}+76204800 \mathrm{R}^{5} \sin (\phi)^{5}+5588352000 \mathrm{R}^{4} \cos (\phi)^{3} \sin (\phi)-5588352000 \mathrm{R}^{4} \cos (\phi) \sin (\phi)^{3}$

$\left.+50295168000 \mathrm{R}^{3} \cos (\phi)^{2} \sin (\phi)-16765056000 \mathrm{R}^{3} \sin (\phi)^{3}+261534873600 \mathrm{R}^{2} \cos (\phi) \sin (\phi)+610248038400 \mathrm{R} \sin (\phi)\right)^{2}$

$-\left(6773760 \mathrm{R}^{7} \cos (\phi)^{5} \sin (\phi)^{2}-11289600 \mathrm{R}^{7} \cos (\phi)^{3} \sin (\phi)^{4}+2257920 \mathrm{R}^{7} \cos (\phi) \sin (\phi)^{6}-152409600 \mathrm{R}^{6} \cos (\phi)^{4} \sin (\phi)^{2}\right.$

$+152409600 \mathrm{R}^{6} \cos (\phi)^{2} \sin (\phi)^{4}+2032128000 \mathrm{R}^{5} \cos (\phi)^{3} \sin (\phi)^{2}-1016064000 \mathrm{R}^{5} \cos (\phi) \sin (\phi)^{4}-16765056000 \mathrm{R}^{4} \cos (\phi)^{2} \sin (\phi)^{2}$

$+80472268800 \mathrm{R}^{3} \cos (\phi) \sin (\phi)^{2}-141120 \mathrm{R}^{8} \cos (\phi)^{6} \sin (\phi)^{2}+352800 \mathrm{R}^{8} \cos (\phi)^{4} \sin (\phi)^{4}-141120 \mathrm{R}^{8} \cos (\phi)^{2} \sin (\phi)^{6}$

$-697426329600 R \cos (\phi)-322560 R^{7} \cos (\phi)^{7}+10160640 R^{6} \cos (\phi)^{6}-10160640 R^{6} \sin (\phi)^{6}-203212800 R^{5} \cos (\phi)^{5}$

$+2794176000 R^{4} \cos (\phi)^{4}+2794176000 R^{4} \sin (\phi)^{4}-26824089600 R^{3} \cos (\phi)^{3}+174356582400 R^{2} \cos (\phi)^{2}-174356582400 R^{2} \sin (\phi)^{2}$

$\left.+5040 \mathrm{R}^{8} \cos (\phi)^{8}+5040 \mathrm{R}^{8} \sin (\phi)^{8}+1307674368000\right)^{2}-\left(40320 \mathrm{R}^{8} \cos (\phi)^{7} \sin (\phi)-282240 \mathrm{R}^{8} \cos (\phi)^{5} \sin (\phi)^{3}+282240 \mathrm{R}^{8} \cos (\phi)^{3} \sin (\phi)^{5}\right.$

$-40320 \mathrm{R}^{8} \cos (\phi) \sin (\phi)^{7}-2257920 \mathrm{R}^{7} \cos (\phi)^{6} \sin (\phi)+11289600 \mathrm{R}^{7} \cos (\phi)^{4} \sin (\phi)^{3}-6773760 \mathrm{R}^{7} \cos (\phi)^{2} \sin (\phi)^{\wedge} 5+322560 \mathrm{R}^{7} \sin (\phi)^{7}$

$+60963840 \mathrm{R}^{6} \cos (\phi)^{5} \sin (\phi)-203212800 \mathrm{R}^{6} \cos (\phi)^{3} \sin (\phi)^{3}+60963840 \mathrm{R}^{6} \cos (\phi) \sin (\phi)^{5}-1016064000 \mathrm{R}^{5} \cos (\phi)^{4} \sin (\phi)$

$+2032128000 \mathrm{R}^{5} \cos (\phi)^{2} \sin (\phi)^{3}-203212800 \mathrm{R}^{5} \sin (\phi)^{5}+11176704000 \mathrm{R}^{4} \cos (\phi)^{3} \sin (\phi)-11176704000 \mathrm{R}^{4} \cos (\phi) \sin (\phi)^{3}$

$\left.-80472268800 \mathrm{R}^{3} \cos (\phi)^{2} \sin (\phi)+26824089600 \mathrm{R}^{3} \sin (\phi)^{3}+348713164800 \mathrm{R}^{2} \cos (\phi) \sin (\phi)-697426329600 * \mathrm{R}^{*} \sin (\phi)\right)^{2}$ 
In order to ascertain the region of stability for the stability function (5) above, we need to get values of $\phi$ from 0 through $360^{\circ}$, and the corresponding values of $\mathrm{R}$ from (23), to enable the construction of the Jordan curve. So the following table values were computed with the help of both MATLAB and MAPLE-18 packages

Table 1. Table of values for stability plot

\begin{tabular}{|c|c|c|c|}
\hline THETA & $\mathbf{R}$ & THETA & $\mathbf{R}$ \\
\hline 0 & 61.5 & $5 \mathrm{pi} / 9$ & -24.3 \\
\hline $\mathrm{pi} / 36$ & 61.3 & $7 \mathrm{pi} / 12$ & -28.9 \\
\hline $\mathrm{pi} / 18$ & 60.9 & 11pi/18 & -33.1 \\
\hline $\mathrm{pi} / 12$ & 60.1 & $23 \mathrm{pi} / 36$ & -36.9 \\
\hline $\mathrm{pi} / 9$ & 59.1 & $2 \mathrm{pi} / 3$ & -40.5 \\
\hline $5 \mathrm{pi} / 36$ & 57.8 & $25 \mathrm{pi} / 36$ & -43.8 \\
\hline $\mathrm{pi} / 6$ & 56.1 & 13pi/18 & -46.8 \\
\hline $7 \mathrm{pi} / 36$ & 54.2 & $3 \mathrm{pi} / 4$ & -49.6 \\
\hline $2 \mathrm{pi} / 9$ & 52.0 & $7 \mathrm{pi} / 9$ & -52.0 \\
\hline $\mathrm{pi} / 4$ & 49.6 & $29 \mathrm{pi} / 36$ & -54.2 \\
\hline $5 \mathrm{pi} / 18$ & 46.8 & $5 \mathrm{pi} / 6$ & -56.1 \\
\hline $11 \mathrm{pi} / 36$ & 43.8 & $31 \mathrm{pi} / 36$ & -57.8 \\
\hline $\mathrm{pi} / 3$ & 40.5 & 8pi/9 & -59.1 \\
\hline 13pi/36 & 36.9 & 11pi/12 & -60.1 \\
\hline $7 \mathrm{pi} / 18$ & 33.1 & $17 \mathrm{pi} / 18$ & -60.9 \\
\hline $5 * \mathrm{pi} / 12$ & 28.9 & $35 \mathrm{pi} / 36$ & -61.3 \\
\hline 4pi/9 & 24.3 & pi & -61.5 \\
\hline $17 \mathrm{pi} / 36$ & 19.2 & $37 \mathrm{pi} / 36$ & -61.3 \\
\hline $\mathrm{pi} / 2$ & 0.0 & 19pi/18 & -60.9 \\
\hline 19pi/36 & -19.2 & $13 \mathrm{pi} / 12$ & -60.1 \\
\hline THETA & $\mathbf{R}$ & THETA & $\mathbf{R}$ \\
\hline $10 \mathrm{pi} / 9$ & -59.1 & 29pi/18 & 33.1 \\
\hline 41pi/36 & -57.8 & 59pi/36 & 80.4 \\
\hline
\end{tabular}

\begin{tabular}{|c|c|c|c|}
\hline THETA & $\mathbf{R}$ & THETA & $\mathbf{R}$ \\
\hline $7 \mathrm{pi} / 6$ & -56.1 & $5 \mathrm{pi} / 3$ & 89.2 \\
\hline 43pi/36 & -54.2 & 61pi/36 & 97.3 \\
\hline $11 \mathrm{pi} / 9$ & -52.0 & $31 \mathrm{pi} / 18$ & 46.8 \\
\hline $5 \mathrm{pi} / 4$ & -49.6 & $7 \mathrm{pi} / 4$ & 49.6 \\
\hline 23pi/18 & -46.8 & $16 \mathrm{pi} / 9$ & 52.0 \\
\hline 47pi/36 & -43.8 & $65 \mathrm{pi} / 36$ & 54.2 \\
\hline $4 \mathrm{pi} / 3$ & -40.5 & $11 \mathrm{pi} / 6$ & 56.1 \\
\hline 49pi/36 & -36.9 & 67pi/36 & 57.8 \\
\hline $25 \mathrm{pi} / 18$ & -33.1 & $17 \mathrm{pi} / 9$ & 59.1 \\
\hline $17 \mathrm{pi} / 12$ & -28.9 & 23pi/12 & 60.1 \\
\hline $13 \mathrm{pi} / 9$ & -24.3 & 35pi/18 & 60.9 \\
\hline $53 \mathrm{pi} / 36$ & -19.2 & $71 \mathrm{pi} / 36$ & 61.3 \\
\hline $3 \mathrm{pi} / 2$ & 0.0 & $2 \mathrm{pi}$ & 61.5 \\
\hline $55 \mathrm{pi} / 36$ & 19.2 & & \\
\hline THETA & $\mathbf{R}$ & THETA & $\overline{\mathbf{R}}$ \\
\hline $14 \mathrm{pi} / 9$ & 24.3 & & \\
\hline 19pi/12 & 28.9 & & \\
\hline
\end{tabular}

This is a clear indication that the above stability (Polar) curve is A-stable, with the regions of stability and instability showing in the curve.

Definition 1: A one - step numerical method is said to be $\mathrm{L}$ - stable if it is A - stable and, in addition, when applied to the test equation $y^{\prime}=\lambda y$, where $\lambda$ is a complex constant with $\operatorname{Re}(\lambda)<0 \quad, \quad$ it yields $\quad y_{n+1}=R_{L, M}(h \lambda) \quad$, where $\lim _{R e(\lambda h) \rightarrow \infty} R_{L, M}(h \lambda)=0$.

So from above definition, further analysis of the stability function (1) above, given as:

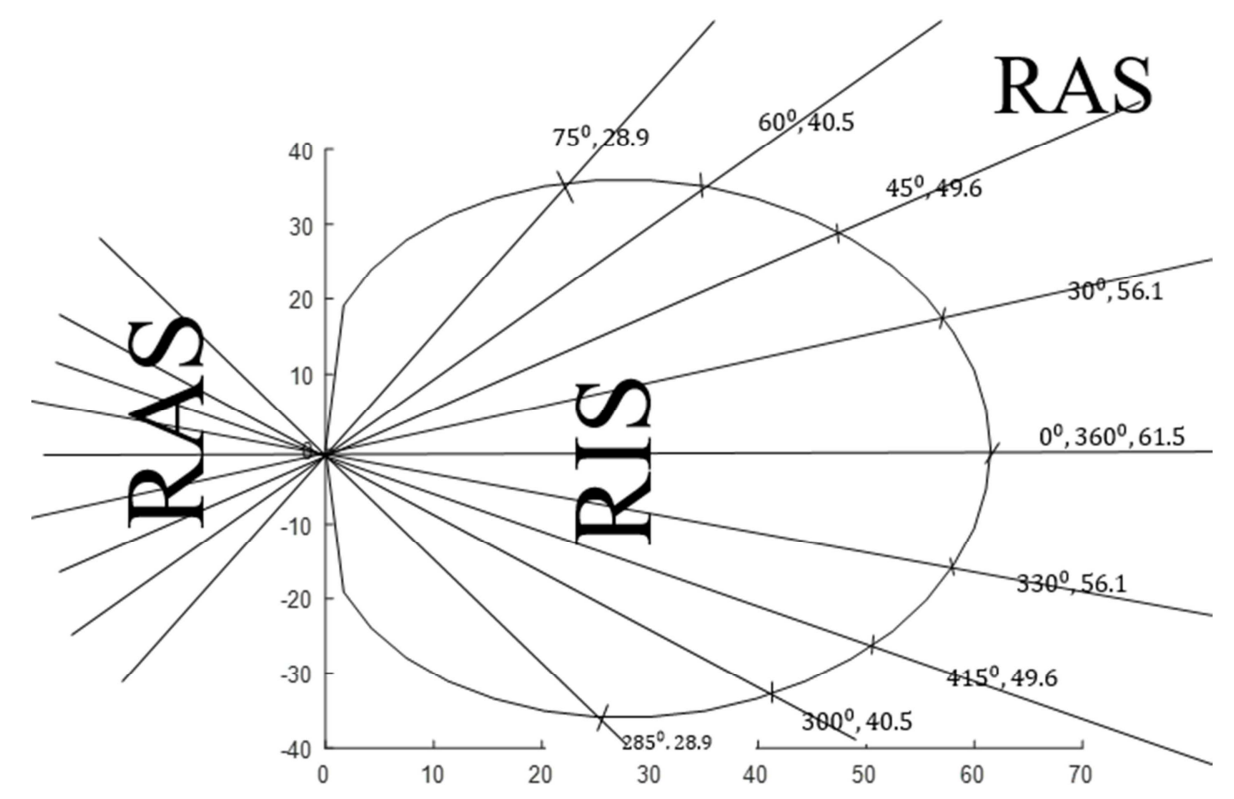

Figure 1. The Jordan curve for $k=8$.

$$
\operatorname{Lim}_{\bar{h} \rightarrow \infty}\left(\frac{\left(8 \overline{\mathrm{h}}^{7}+504 \overline{\mathrm{h}}^{6}+15120 \overline{\mathrm{h}}^{5}+277200 \overline{\mathrm{h}}^{4}+3326400 \overline{\mathrm{h}}^{3}+25945920 \overline{\mathrm{h}}^{2}+121080960 \overline{\mathrm{h}}+259459200\right)}{\left(\overline{\mathrm{h}}^{8}-64 \overline{\mathrm{h}}^{7}+2016 \overline{\mathrm{h}}^{6}-40320 \overline{\mathrm{h}}^{5}+554400 \overline{\mathrm{h}}^{4}-5322240 \overline{\mathrm{h}}^{3}+34594560 \overline{\mathrm{h}}^{2}-138378240 \overline{\mathrm{h}}+259459200\right)}\right)=0
$$

Which further shows that the rational integrator scheme is L-Stable. 


\section{Discussion}

After a successful expansion of the rational integrator, we represented the integrating function in the form of polar curve using MATLAB and MAPLE-18 packages. Thus, the following findings are visible.

1) It shows the region of absolute stability

2) The exterior (outside) of the curve represent the region of absolute stability (RAS) of the integrator.

3) The Region of instability (RIS) is on the positive side of the complex plane, which is within the stability (polar) curve.

4) The rational integrator $\tau$ is within the interval \pm 61.48147892 where $\tau$ is the encroachment point.

\section{Conclusion}

It can be concluded that the Region of Absolute Stability (RAS) of the rational Interpolation method lies entirely on the Left-half of the complex plane. At the same time, the region of instability (RIS), lies within the Jordan curve as seen from the figure 1 above. This work revealed that, the Region of Absolute Stability (RAS) for $\mathrm{k}=8$ is a superset of the entire left-half of the complex plane. Furthermore, the rational interpolation scheme is not only A-stable, but also L- stable.

\section{References}

[1] Aashikpelokhai U. S. U. (2000). A variable order numerical integrator based on rational interpolants. Journal of Nig. Mathematical Society. Vol. 19, 27-28.

[2] Aashikpelokhai U. S. U and Momodu I. B. A (2006): "A high order rational integrator for stiff systems" Journal of Nigeria Annuals of Natural Sciences pg. 122-143, vol. 4 (1).

[3] Elakhe A. O., Onianwa C. U. and Elakhe S. O. (2016). A new order five numerical rational Rational Integrator. Aiziza Journal of Science and Technology, Vol. 1, pp49-63.

[4] Agbeboh, G. U. (2006). Comparison of some one-step integrator for solving singular initial value problems. Ph. D. Thesis, AAU
[5] Abhulimen, C. E. and Otunta, F. O. (2007): A Family of Two Step Exponentially Fitted Multiderivable Methods for the Numerical Integration of Stiff IVPs in ODE. International Journal of Numerical Mathematics, Vol. 2, pp1-21.

[6] Agbeboh, G. U. and Aashikpelokhai U.S.U. (2002). An analysis of order, thirteen rational integrator. Journal of Sci. Engr. Tech Vol. 9 (2), 4128-4145.

[7] Aashikpelokhai U. S. U. and Elakhe, A. O. (2010). A moderate order numerical integrator for still differential system, Journal of Nigeria Association of Mathematical Physics. Vol. 17. Pp. 425-432.

[8] Islam, M. A. (2015). A Comparative Study on Numerical Solutions of Initial Value Problems (IVP) for Ordinary Differential Equations (ODE) with Euler and Runge-Kutta Methods. American Journal of Computational Mathematics, Vol. 5, 393-404.

[9] Agbeboh, G. U. Esekhaigbe, C. A. (2016): Transformation and Implementation of a Highly Efficient Fully Implicit Fourth-Order Runge-Kutta Method. International Journal of Innovative Research, Vol 5 (1), 171-183.

[10] Elakhe, A. O. and Aashikpelokhai U.S.U (2013): Singulo Oscillatory - Stiff rational integrators. International Journal of Physical Sciences, vol. 8 (34), 1703-1715.

[11] Fatunla S. O. and Aashikpelokhai, U.S.U (1994). A Fifth Order L-Stable Numerical Integrator, Scientific Comp pp 68-86.

[12] Elakhe, A. O., Aashikpelokhai U.S.U. and Ebhomien P. A. (2011): A Dynamical Singulo-Stiff Rational Integrator. IRCAB Journal of Natural Applied Sciences. Vol. 1 pp73-79.

[13] Awari, Y. S. (2017): Numerical Strategies for the System of First Order IVPs Using Block Hybrid Extended Trapezoidal Multistep Method of Second Kind for Stiff ODEs. Science Journal of Applied Mathematics and Statistics, Vol. 5 (5): 181-187.

[14] Momodu, I. B. A. (2006): Solution of Singulo-stiff Ordinary Differential Equation. PhD Thesis, AAU, Ekpoma.

[15] Fatunla, S. O. (1978): An Implicit Two-Part Numerical Integration Formula for Linear and Non-Linear Stiff System of ODE”, Mathematics of Computation 32, 1-11. 